This material is posted here with permission of the IEEE. Such permission of the IEEE does not in any way imply IEEE endorsement of any of Helsinki University of Technology's products or services. Internal or personal use of this material is permitted. However, permission to reprint/republish this material for advertising or promotional purposes or for creating new collective works for resale or redistribution must be obtained from the IEEE by writing to pubs-permissions@ieee.org.

By choosing to view this document, you agree to all provisions of the copyright laws protecting it. 


\title{
Effect of Reflections on Nonstationary Gyrotron Oscillations
}

\author{
Markus I. Airila and Pia Kåll
}

\begin{abstract}
We present an extension of the self-consistent timedependent theory describing nonstationary processes in gyrotrons to allow for reflections. Different mathematical descriptions of partial reflection of the output signal are compared, and numerical algorithms for analyzing them are given. Using a novel description, we have computed a map of gyrotron oscillations, which identifies the regimes of stationary, periodically modulated and chaotic oscillations in the plane of generalized gyrotron variables when reflection is present. In general, reflections drive the gyrotron into quasiperiodic oscillations instead of chaos, but also the threshold current for chaotic oscillations decreases somewhat. The results can be exploited in the development of high-power gyrotrons for electron cyclotron resonance heating (ECRH) and electron cyclotron current drive (ECCD) of fusion plasmas, but also in low-power applications, where chaotic oscillations might be useful.
\end{abstract}

Index Terms-Gyrotron, reflections, quasiperiodicity, chaos

\section{INTRODUCTION}

$\mathbf{S}$ EVERAL theoretical studies have predicted that gyrotrons can generate periodically modulated and chaotic oscillations in addition to stationary ones. The regimes of different types of oscillations form a complicated map in the operating parameter plane. In general, nonstationary oscillations are more likely to appear at higher values of the beam current, and, indeed, the onset of chaotic oscillations requires a much larger current than is routinely used in present high-power high-frequency gyrotrons. [1]-[4] These theoretical findings are supported by recent experimental results [5].

Fusion energy research demands increasingly powerful high-frequency gyrotrons for plasma heating and current drive. Such tubes should provide a stable high-power signal at a well-defined frequency, which requires operation in the region of stationary oscillations and high efficiency. On the other hand, many applications in physics, industry, and even medicine would take advantage of the bandwidth broadening resulting from chaotic oscillations: e.g., significantly better uniformity of volumetric microwave heating can be achieved using waves with a broadband spectrum. Although stationarity of microwave oscillations seems to be characteristic for highefficiency operation of an ideal (reflectionless) tube [4], it has been found that reflection of microwaves from the window, the load, or output-section inhomogeneities could lower the

Manuscript received April 10, 2003; revised June 27, 2003. The work of M.A. was supported by the KAUTE foundation, the Jenny and Antti Wihuri foundation, and the Finnish Foundation for Promotion of Technology.

The authors are with Helsinki University of Technology, EuratomTekes Association, FIN-02015 HUT, Finland (e-mail: markus.airila@hut.fi, pia.kall@hut.fi). threshold for stochastic oscillations even to one tenth of its unperturbed value [6], [7], negatively influence mode competition [8], [9], and give rise to frequency jumps during pulses which heat the cavity [10]. Recently it has been suggested on the basis of numerical simulations [11], [12] that reflections make a gyrotron show complicated quasiperiodic behavior rather than oscillate chaotically. In any case reflections may pose a limitation for the attempts to increase output power using higher beam current or, on the other hand, make it possible to achieve desired bandwidth broadening without raising the current unrealistically high.

In this paper we discuss different reflection models for numerical work and report self-consistent calculations of the temporal behavior of gyrotron oscillations in the case of partial reflection of the signal at the output. Our work is based on the description of gyrotron interaction given in [1], which assumes that the transversal structure of the high-frequency field is fixed but the field amplitude is allowed to vary in the axial direction. In our calculations reflection is taken into account by artificially launching a backward-traveling wave representing the delayed reflected signal. The main results of our computations are given as a detailed map covering the most relevant part in the plane of the generalized gyrotron variables: cyclotron resonance mismatch and dimensionless current. In addition, we analyze output signals to distinguish between chaos and quasiperiodicity, and show that our model is consistent with a description valid for stationary cases. Compared to previous studies of nonstationary oscillations with allowance for reflections, the present approach is rather general by nature, since we have worked with the simplest model for the resonator-cylindrical—and made no assumptions about the cause of reflection. The reflection is described by the reflection coefficient $R$ and the delay time $T$.

\section{Mathematical Reflection MOdels}

\section{A. Gyrotron equations without reflection}

To describe self-consistently nonstationary gyrotron oscillations we use the system of partial differential equations derived in [1]:

$$
\begin{aligned}
\frac{d p}{d \zeta}+i\left(\Delta+|p|^{2}-1\right) p & =i f(\zeta, \tau) \\
\frac{\partial^{2} f}{\partial \zeta^{2}}-i \frac{\partial f}{\partial \tau}+\delta f & =\frac{I}{2 \pi} \int_{0}^{2 \pi} p d \vartheta_{0}
\end{aligned}
$$

where $p$ is the complex transverse momentum of the electron normalized to its initial absolute value, $\zeta=\left(\beta_{\perp 0}^{2} \omega_{\mathrm{c}} / 2 \beta_{\| 0} c\right) z$ 
is the dimensionless coordinate $\left(0 \leq \zeta \leq \zeta_{\text {out }}\right), \beta_{\perp 0}=v_{\perp 0} / c$ and $\beta_{\| 0}=v_{\| 0} / c$ are normalized electron velocities, $\Delta=$ $2\left(\bar{\omega}-\omega_{\mathrm{c}}\right) / \beta_{\perp 0}^{2} \bar{\omega}$ is the frequency mismatch, $\omega_{\mathrm{c}}$ is the electron cyclotron frequency, $f(\zeta)$ is the axial profile of the highfrequency field amplitude in the resonator, $\tau=\frac{1}{8} \beta_{\perp 0}^{4} \beta_{\| 0}^{-2} \bar{\omega} t$ is the dimensionless time, $\delta=8 \beta_{\| 0}^{2} \beta_{\perp 0}^{-4}[1-\omega(\zeta) / \bar{\omega}]$ describes variation of the critical frequency $\omega(\zeta)$ along the resonator axis, $\bar{\omega}$ is the cut-off frequency at the exit from the resonator, and $I$ is the dimensionless current. This description is valid for operation at the fundamental cyclotron resonance.

The system of equations (1)-(2) has to be supplemented by the standard initial condition for the momentum, $p(0)=$ $\exp \left(i \vartheta_{0}\right)$ with $0 \leq \vartheta_{0}<2 \pi$, and by the boundary condition for the field at the entrance to the interaction space:

$$
f(0, \tau)=0,
$$

which means that the wave energy cannot propagate into the input taper. The ideal case without reflection is obtained using the reflectionless boundary condition at the exit from the interaction space:

$$
f\left(\zeta_{\text {out }}, \tau\right)+K\left\{f(\zeta, \tau) ; \zeta_{\text {out }}\right\}=0
$$

with a shorthand notation

$$
\left.K\left\{f(\zeta, \tau) ; \zeta_{0}\right\} \equiv \frac{1}{\sqrt{\pi i}} \int_{0}^{\tau} \frac{1}{\sqrt{\tau-\tau^{\prime}}} \frac{\partial f\left(\zeta, \tau^{\prime}\right)}{\partial \zeta} d \tau^{\prime}\right|_{\zeta=\zeta_{0}} .
$$

Finally, an initial condition for the field profile is needed. We started the computations with a field

$$
f(\zeta, 0)=0.1 \sin \left(\pi \frac{\zeta}{\zeta_{\text {out }}}\right),
$$

which simulates an axial field profile with only one maximum.

The electron perpendicular efficiency $\eta_{\perp}$ which describes the extraction of the electron orbital momentum from the beam is given by the expression

$$
\eta_{\perp}=1-\frac{1}{2 \pi} \int_{0}^{2 \pi}\left|p\left(\zeta_{\text {out }}\right)\right|^{2} d \vartheta_{0}
$$

and can be calculated at any time when the field profile is known.

Reflections should be added to this formulation in a way that allows flexible adjustment of all significant parameters characterizing reflection. In the following we discuss several alternatives. Fig. 1 illustrates how the gyrotron resonator is described in these models. In (a), corresponding to the reflectionless case, just the interaction cavity has been sketched, and the generated wave exits the resonator in its entirety. The helical electron trajectories depict the beam interacting with the RF field.

\section{B. Geometrically induced reflection}

Reflections can be induced by defining a narrowing (an iris) at the output section with the help of the function $\delta(\zeta)$ appearing in Eq. (2), as illustrated in Fig. 1(b). The critical frequency is inversely proportional to the cavity radius, so that

$$
\delta(\zeta)=\frac{8 \beta_{\| 0}^{2}}{\beta_{\perp 0}^{4}}\left(1-\frac{\omega(\zeta)}{\bar{\omega}}\right)=\frac{8 \beta_{\| 0}^{2}}{\beta_{\perp 0}^{4}}\left(1-\frac{R_{0}}{R(\zeta)}\right),
$$

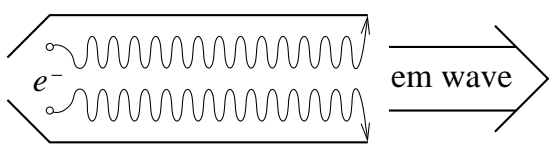

a) no reflection

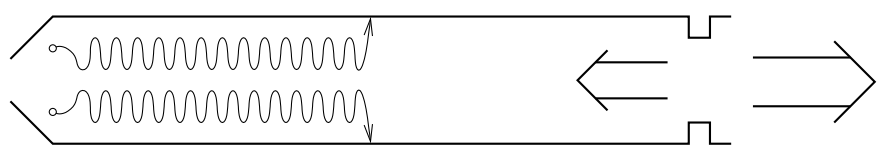

b) reflection by geometric obstacles

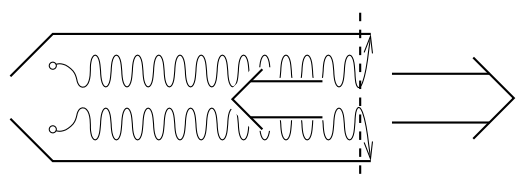

c) reflection by boundary condition; no delay

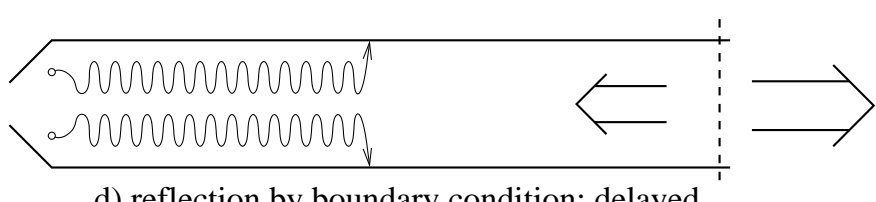

d) reflection by boundary condition; delayed

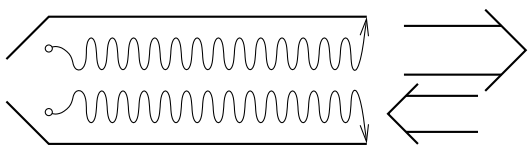

e) external reflected wave

Fig. 1. Illustration of different ways to describe reflections in gyrotrons. (a) In the reflectionless case the whole electromagnetic wave exits the resonator. (b) Reflections were modeled in [7] with the help of an iris located at a distance comparable to the length of the interaction space. (c) A reflective boundary condition can be applied at the resonator end, using the phase of the reflection coefficient to simulate delay. (d) The iris can be replaced by the reflective boundary condition at the same location. (e) The reflected wave can be actively launched after the delay time $T$.

where $R_{0}$ stands for the cavity radius at the exit and $R(\zeta)$ differs from $R_{0}$ only at the iris. The reflection is delayed by extending the waveguide beyond the resonator: Eq. (2) is solved for $f$ in the whole region $\zeta \in\left[0, \zeta_{\text {iris }}\right]$, but the current term is set to zero for $\zeta>\zeta_{\text {out }}$, signifying the fact that beam-wave interaction takes place in the resonator only. The dimensionless cavity length $\zeta_{\text {out }}$ in real gyrotrons is usually around 15. At the exit boundary behind the iris one can use the reflectionless boundary condition. Using this approach with $\zeta_{\text {iris }} \approx 40$, stochastic oscillations were found in [7] with operating parameters normally corresponding to a stationary output signal.

In numerical solution of Eqs. (1)-(2), the grid of discrete coordinate values must be extended all the way to the iris, which makes it impractical to study reflections from distant components (about one resonator length, a few centimeters, is still tolerable). Since we wish to simulate reflections taking place further away, this model turns out to be inappropriate. Also, as we aim at a general map of gyrotron oscillations in the presence of reflections, a description in terms of the general 
reflection parameters $R$ and $T$ is preferred to any one using geometric parameters (as the position, length and depth of the iris).

\section{Reflective boundary condition}

Alternatively, reflection can be taken into account by the normalized boundary condition at the exit from the interaction space:

$$
\operatorname{Re}^{i \rho}=\frac{f\left(\zeta_{\text {out }}, \tau\right)+K\left\{f(\zeta, \tau) ; \zeta_{\text {out }}\right\}}{f\left(\zeta_{\text {out }}, \tau\right)-K\left\{f(\zeta, \tau) ; \zeta_{\text {out }}\right\}}
$$

where $R$ is the absolute value of the reflection coefficient and $\rho$ is the phase shift in the reflection [see Fig. 1(c)]. For $R=$ 0 this boundary condition reduces to the reflectionless case. Since the energy density of the field is proportional to the square of the field amplitude, the fraction of power reflection is $R^{2}$.

In order to minimize the number of parameters characterizing reflection, one can simulate reflection delay by altering the phase shift $\rho$. The phase shift corresponding to a delay $T$ is simply $\rho=\bar{\omega} T+\rho^{\prime}$, where the shift $\rho^{\prime}$ occurs instantaneously at the reflection point. This approach is very general: there remains only one (complex) parameter characterizing the reflection. For practical application of the results one must estimate $R$ and $\rho$ in the specific case of interest (see, e. g., [8]). However, all features of delayed reflection can be reproduced in this way only if the output signal is stationary, as in [13]. Instead, when nonstationary processes are considered, also the amplitude modulation plays a role. This fact should enter the model as well: a delayed amplitude of the reflected wave must be used so that artificial correlation between the amplitudes of the reflected and resonator fields is avoided.

Amplitude modulation of the delayed signal can be properly taken into account by introducing a variant of the model [see Fig. 1(d)]. This alternative is obtained by combining the description presented above with the model of Sec. IIB: just replace the iris with the reflective boundary condition at the same position. While the reflected wave can be modeled truly delayed this way, the limitations caused by the extended computation domain remain.

\section{Three-wave model}

Fig. 1(e) illustrates the model which we chose for this study and which allows us to perform all computations between $\zeta=0$ and $\zeta=\zeta_{\text {out }}$. The limitations in the size of the computation grid can be circumvented by treating the reflected wave $f_{\mathrm{R}}$ and the resonator field (here called $f_{0}$ ) as separate quantities and launching $f_{\mathrm{R}}$ only after the delay time $T$. In regard to the role of the phase shift $\rho$ in determining the behavior of the system, we refer to the discussion given in [12]. First, it was demonstrated there that variations of the distance to the reflecting load in the length scale comparable to this distance itself certainly have a significant effect on the oscillations. This results from the fact that the delay time and the eigenfrequencies change remarkably. On the other hand, small variations of the distance (of the order of half wavelength) also somewhat affect the radiation spectra but have no influence on the qualitative behavior. It can be concluded that the exact phase difference between the reflected and resonator fields is responsible for the small changes, but the parameter $\rho^{\prime}$ (having a value between 0 and $2 \pi$ and thus constituting a negligible fraction of the total phase shift $\rho$ ) cannot be used to switch the system between stationary, periodic, and chaotic states. We (somewhat arbitrarily) set $\rho^{\prime}$ to a value representing total reflection, $\rho^{\prime}=\pi$, which determines $\rho$ through the relation $\rho=\bar{\omega} T+\rho^{\prime}$, and regarded $R$ and $T$ as the significant variable parameters characterizing reflection.

We now describe how our model is mathematically formulated. Assume that the outgoing wave is partly reflected by an obstacle located at $z=z_{\text {out }}+z_{0}$. Then the reflected part, traveling at the velocity of light, reaches the resonator end again after the dimensionless time

$$
T=\frac{\beta_{\perp 0}^{4} \bar{\omega} z_{0}}{4 \beta_{\|}^{2} c} .
$$

We simulate this with a new wave whose amplitude is $R$ times the amplitude of the outgoing wave at the time moment $\tau-T$. After entering the resonator at the output crosssection, the reflected wave travels backwards and experiences another (total) reflection at the input tapering. This is most conveniently formulated by dividing the reflected wave further into backward- and forward-propagating parts $f_{\mathrm{R} 1}$ and $f_{\mathrm{R} 2}$, respectively. Due to the co-existence of three waves, the electrons now obey the equation of motion

$\frac{d p}{d \zeta}+i\left(\Delta+|p|^{2}-1\right) p=i\left[f_{0}(\zeta, \tau)+f_{\mathrm{R} 1}(\zeta, \tau)+f_{\mathrm{R} 2}(\zeta, \tau)\right]$

and the evolution of the fields in a cylindrical resonator $[\delta(\zeta) \equiv 0]$ is obtained from

$$
\begin{aligned}
\frac{\partial^{2} f_{0}}{\partial \zeta^{2}}-i \frac{\partial f_{0}}{\partial \tau} & =\frac{I}{2 \pi} \int_{0}^{2 \pi} p d \vartheta_{0}, \\
\frac{\partial^{2} f_{\mathrm{R} 1}}{\partial \zeta^{2}}-i \frac{\partial f_{\mathrm{R} 1}}{\partial \tau} & =0, \\
\frac{\partial^{2} f_{\mathrm{R} 2}}{\partial \zeta^{2}}-i \frac{\partial f_{\mathrm{R} 2}}{\partial \tau} & =0,
\end{aligned}
$$

i. e., the beam current acts as the source of $f_{0}$ while the reflected waves travel freely in the resonator.

The initial and boundary conditions for $f_{0}$ and $p$ are the same as in the reflectionless model of nonstationary oscillations. The reflected waves, instead, do not exist at all when $\tau<T$, so the initial condition for them is

$$
f_{\mathrm{R} 1}(\zeta, 0)=f_{\mathrm{R} 2}(\zeta, 0)=0 .
$$

The backward-wave $f_{\mathrm{R} 1}$ is launched using the boundary condition

$$
f_{\mathrm{R} 1}\left(\zeta_{\text {out }}, \tau\right)=-R\left[f_{0}\left(\zeta_{\text {out }}, \tau-T\right)+f_{\mathrm{R} 2}\left(\zeta_{\text {out }}, \tau-T\right)\right] .
$$

We take into account multiple reflections with the $f_{\mathrm{R} 2}$ term, and our calculations are therefore not limited to small $R$. In the reflection at the input taper, $\zeta=0$, the backward-wave $f_{\mathrm{R} 1}$ becomes forward-propagating again and is since then described by $f_{\mathrm{R} 2}$ alone. Otherwise the boundary condition (16) 
would later cause unphysical reflection at $\zeta=\zeta_{\text {out }}$. In order to convert $f_{\mathrm{R} 1}$ into $f_{\mathrm{R} 2}$, we used as a mathematical trick the reflectionless boundary condition at $\zeta=0$, that is,

$$
f_{\mathrm{R} 1}(0, \tau)-K\left\{f_{\mathrm{R} 1}(\zeta, \tau) ; 0\right\}=0 .
$$

This is simply a means of preventing $f_{\mathrm{R} 1}$ from having a forward-propagating component and should not be interpreted as a statement that the wave travels into the beam tunnel. The requirement that also the total field of the reflected wave $f_{\mathrm{R} 1}+f_{\mathrm{R} 2}$ should vanish at $\zeta=0$ can now be fulfilled by exciting the wave $f_{\mathrm{R} 2}$ with a boundary condition describing total reflection

$$
f_{\mathrm{R} 2}(0, \tau)=-f_{\mathrm{R} 1}(0, \tau) .
$$

Finally, we let the wave $f_{\mathrm{R} 2}$ exit the resonator without further reflection:

$$
f_{\mathrm{R} 2}\left(\zeta_{\text {out }}, \tau\right)+K\left\{f_{\mathrm{R} 2}(\zeta, \tau) ; \zeta_{\text {out }}\right\}=0 .
$$

Now Eqs. (11)-(14) as well as the corresponding initial and boundary conditions together are equivalent to Eqs. (1)-(2) with the exception that the incoming wave is actively launched inwards at $\zeta=\zeta_{\text {out }}$.

\section{COMPutations}

The finite difference scheme used in [4] is perfectly suitable for numerical solution of the equations for all reflection models presented in the previous section. The form of the equations for $f$ remains the same in all cases, so that it is the discrete counterpart of (2),

$\frac{1}{h^{2}} f_{j+1}^{n+1}-\left(\frac{2}{h^{2}}+\frac{i}{\Delta \tau}+\delta_{j}\right) f_{j}^{n+1}+\frac{1}{h^{2}} f_{j-1}^{n+1}=g_{j}^{n}-\frac{i}{\Delta \tau} f_{j}^{n}$,

for $j=1, \ldots, N-1$, which is to be solved at each time step. Here $h$ is the spatial step, $\Delta \tau$ the time step, $f_{j}^{n}=f(j h, n \Delta \tau)$, and the discretized current term

$$
g_{j}^{n}=\sum_{k=0}^{s} \frac{I p\left(j h, n \Delta \tau, k \Delta \vartheta_{0}\right)}{s}
$$

is obtained by solving the equation of motion for electrons using the known field profile $f^{n}$ and a fourth-order RungeKutta integration routine. Different descriptions of reflection lead to different $\delta_{j}, g_{j}^{n}$, and $N$. Also the boundary conditions for each model are formulated separately. The resulting linear system of equations remains tridiagonal, whichever model is used. To solve it, we used the routine given in [14] after generalizing it to complex variables.

When an iris is modeled at the output cross-section, the computation region is extended by increasing the number of grid points (to $N^{\prime}$, say) while keeping the same value of $h$. The boundary conditions are the same as in the reflectionless case; they can be written

$$
f_{0}^{n+1}=0
$$

and

$$
\begin{array}{r}
\left.-2 \sqrt{n+1}+\sum_{j=0}^{n} \frac{1}{\sqrt{n+1-j}}\right) f_{N^{\prime}-1}^{n+1} \\
+\left(\frac{h \sqrt{\pi i}}{\sqrt{\Delta \tau}}+2 \sqrt{n+1}-\sum_{j=0}^{n} \frac{1}{\sqrt{n+1-j}}\right) f_{N^{\prime}}^{n+1} \\
=\sum_{j=0}^{n} \frac{f_{N^{\prime}-1}^{j}-f_{N^{\prime}}^{j}}{\sqrt{n+1-j}} .
\end{array}
$$

At the location of the iris, $\delta_{j}$ is let differ from zero according to (8). The absence of beam-wave interaction outside the resonator is taken into account by setting $g_{j}^{n}=0$ for $N<$ $j \leq N^{\prime}$.

As far as the description of reflection with the help of the reflective boundary condition (9) is concerned, the right-hand boundary condition is written in discrete form as

$$
\begin{gathered}
\left(1+R e^{i \rho}\right)\left[-2 \sqrt{n+1}+\sum_{j=0}^{n} \frac{1}{\sqrt{n+1-j}}\right] f_{N-1}^{n+1} \\
+\left[\left(1-R e^{i \rho}\right) \frac{h \sqrt{\pi i}}{\sqrt{\Delta \tau}}\right. \\
\left.+\left(1+R e^{i \rho}\right)\left(2 \sqrt{n+1}-\sum_{j=0}^{n} \frac{1}{\sqrt{n+1-j}}\right)\right] f_{N}^{n+1} \\
=\left(1+R e^{i \rho}\right) \sum_{j=0}^{n} \frac{f_{N-1}^{j}-f_{N}^{j}}{\sqrt{n+1-j}} .
\end{gathered}
$$

Finally, we write out the discretized boundary conditions corresponding to (16), (17), and (18) for the reflected waves of the three-wave model:

$$
f_{\mathrm{R} 1, N}^{n+1}=-R\left[f_{0, N}^{n+1-T / \Delta \tau}+f_{\mathrm{R} 2, N}^{n+1-T / \Delta \tau}\right],
$$

$$
\begin{aligned}
& \left(2 \sqrt{n+1}-\sum_{j=0}^{n} \frac{1}{\sqrt{n+1-j}}\right) f_{\mathrm{R} 1,0}^{n+1} \\
& +\left(\frac{h \sqrt{\pi i}}{\sqrt{\Delta \tau}}-2 \sqrt{n+1}+\sum_{j=0}^{n} \frac{1}{\sqrt{n+1-j}}\right) f_{\mathrm{R} 1,1}^{n+1} \\
& =\sum_{j=0}^{n} \frac{f_{\mathrm{R} 1,1}^{j}-f_{\mathrm{R} 1,0}^{j}}{\sqrt{n+1-j}},
\end{aligned}
$$

and

$$
f_{\mathrm{R} 2,0}^{n+1}=-f_{\mathrm{R} 1,0}^{n+1},
$$

the three boundary conditions not mentioned here being similar to those in the reflectionless case.

In the calculations we used $\zeta_{\text {out }}=15$, which is close to the optimum with respect to efficiency. The spatial step length was $h=0.25$, and the time step was increased from $\Delta \tau=$ 0.02 (used in [4]) to $\Delta \tau=0.1$, since the region $\Delta<0$, where the solutions vary rapidly in time, was left outside of the present study. To give some idea about the magnitudes of the dimensionless time scale, we give corresponding physical 
delay time and modulation frequencies assuming the operating frequency to be $\bar{\omega} / 2 \pi=170 \mathrm{GHz}$, accelerating voltage $U=$ $90 \mathrm{kV}$, and the pitch factor $\alpha=1.3$.

Adding reflections to the system, we give rise to a new time scale defined by the delay $T$. This means that the initial transient phase of the oscillations becomes remarkably longer in comparison to the reflectionless case. Therefore, also much longer computations are needed for finding out the type of oscillations of the gyrotron. Instead of $\tau_{\text {end }}=500\left(t_{\text {end }} \approx\right.$ $13 \mathrm{~ns}$ ) used in [4], we extended the numerical solution of the Eqs. (1)-(2) up to $\tau_{\text {end }}=15000$ ( $t_{\text {end }} \approx 380 \mathrm{~ns}$ ) to ensure that the oscillations have attained their final state.

\section{RESUlTS}

As an illustration of the effect of reflections, we show in Figs. 2-4 three different output signals (a) together with their Fourier spectra (b) and autocorrelation functions (c). These signals have been obtained using parameter values $\Delta=0.20$ and $I=0.10$; this point belongs to the stationary region of Fig. 1 in [4] [reproduced here as Fig. 6(a)]. The choice of reflection parameters was done as follows: We chose to simulate reflections from the gyrotron window and took therefore $T=200$, which corresponds to reflection at a distance of about $0.8 \mathrm{~m}$ from the cavity with our assumptions about the gyrotron (see above). The reflection coefficient $R$ was then varied so that the different types of oscillations were found. Up to $R=0.20$ the oscillations remain stationarybeyond that value, nonstationary phenomena appear.

Fig. 2 shows periodic oscillations resulting from $R=0.25$ (power reflection 6\%). The signal is modulated by a few discrete frequencies that can easily be distinguished in the Fourier spectrum. The dominant modulation frequency corresponds approximately to the back-and-forth transit time of the wave between the input taper and the reflection point. Also the autocorrelation function reflects the fact that the signal repeats itself - it reaches almost unity again and again after several delay times. For $R=0.30$ (power reflection $9 \%$ ), the signal already shows quite strong aperiodicity (Fig. 3). However, there remain two dominant frequencies in the spectrum, and the fundamental period is clearly visible as the maxima of the autocorrelation function. In Fig. 4 we show that the signal becomes chaotic, shows a broad spectrum and has practically no autocorrelation as the reflection coefficient is increased to $R=0.45$ (power reflection $20 \%$ ). In spite of the drastic change in the output signal, generation efficiency decreases only a little with increasing $R$. In the absence of reflections, the time-averaged orbital efficiency $\left\langle\eta_{\perp}\right\rangle$ is $12.4 \%$, the corresponding values for different $R$ being shown in the captions of Figs. 2-4. Finally, to address the question whether reflections really drive the system into chaos and not into a quasiperiodic orbit, we show Fig. 5, which corresponds to $\Delta=0.0, I=0.018$ and the same reflection parameters as in the previous example. Here the signal appears to be aperiodic, but its spectrum reveals that it is to a large extent composed of a few frequencies. This is the most typical case in the parameter region we have studied, and therefore we can confirm the conclusion made in [12] that usually reflection

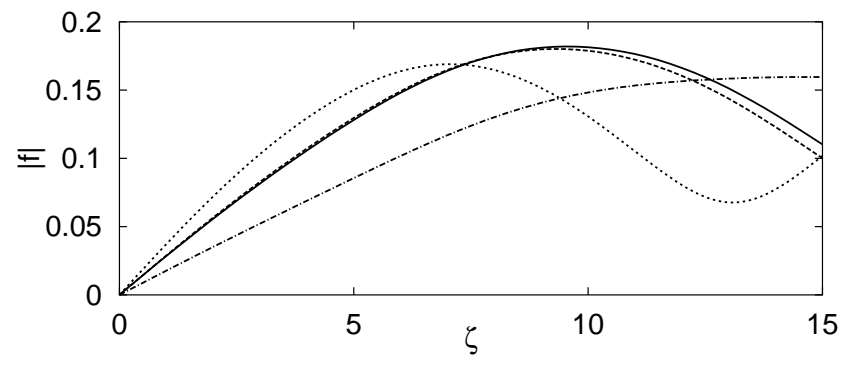

Fig. 7. The stationary field envelope $|f(\zeta)|$ calculated with the three-wave model (solid line) and with the model of Sec. II-C using $\rho=2.25 \mathrm{rad}\left(129^{\circ}\right)$ (dashed line) and $\rho=4.00 \mathrm{rad}\left(229^{\circ}\right)$ (dotted line). Also the field profile corresponding to the reflectionless case is shown (dash-dot). Here $\Delta=0.60$, $I=0.01$

leads to quasiperiodicity instead of chaos. The chaotic case of Fig. 4 probably just indicates the fact that reflection can slightly lower the threshold for chaotic oscillations. Indeed, Fig. 6(a) shows that the point $\Delta=0.20, I=0.1$ is located rather close to the boundary of the chaotic regime even in the absence of reflection.

In order to generalize the "map" of Fig. 6(a), we repeated with reflections the computations presented in [4]. The results are shown as a new map in the operating parameter plane in Fig. 6(b). Due to the increased computation time, we limited our study to the most interesting region in the $(\Delta, I)$ plane: $0 \leq \Delta \leq 0.8,0.003 \leq I \leq 0.1$. This parameter range is almost completely characterized by stationary oscillations if there is no reflection. The reflection coefficient was chosen to be $R=0.45$, which is comparable to the effect of the iris used in [7]. This value is rather high for high-power gyrotrons, but in low-power applications the reflection could be even stronger if introduced on purpose to generate chaotic oscillations. Also here we have taken $T=200$.

To demonstrate that the models of Secs. II-C [reflective boundary condition (9)] and II-D (three-wave) give similar results in a stationary case, we show in Fig. 7 the stationary field profile for $\Delta=0.60, I=0.01$ calculated using both approaches. With these parameter values one obtains the maximum orbital efficiency in the absence of reflections. The phase shift corresponding to $T=200$ was found to be $\rho=2.25 \mathrm{rad}\left(129^{\circ}\right)$ in this case. The profiles agree rather well, but note that by varying $\rho$ in the stationary model, one obtains very different profiles. With $R=0.45$, the perpendicular efficiency $\eta_{\perp}$ decreases from $75 \%$ of the reflectionless case to $45-70 \%$ depending on $\rho$.

In general [compare Fig. 6(b) to Fig. 6(a)], the following effects of reflections on gyrotron operation can be listed:

- The threshold current for chaotic oscillations decreases somewhat due to $20 \%$ power reflection.

- Nonstationary output appears also with parameter values close to the maximum efficiency point.

- Automodulation with a long period (comparable to the delay $T$ ) and quasiperiodic oscillations occur over large regions of the operating parameter plane. 

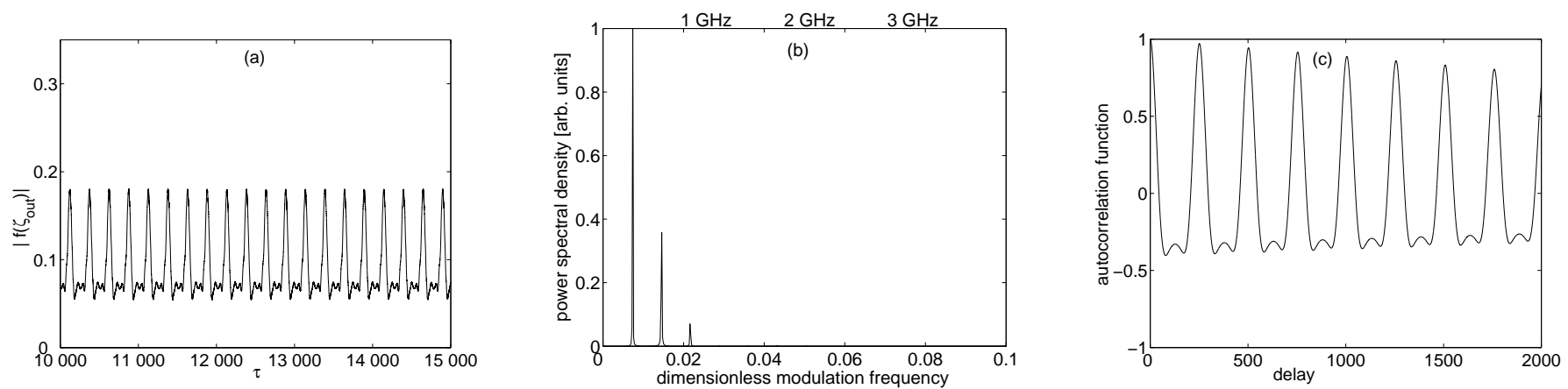

Fig. 2. (a) Gyrotron signal, (b) its power spectrum, and (c) autocorrelation function for $\Delta=0.20, I=0.10, R=0.25$, and $T=200$. The signal $\left|f\left(\zeta_{\text {out }}\right)\right|=\left|f_{0}\left(\zeta_{\text {out }}\right)+f_{\mathrm{R} 2}\left(\zeta_{\text {out }}\right)\right|$ is clearly modulated by a few discrete frequencies and it repeats itself almost perfectly after several delay times. The time-averaged orbital efficiency $\left\langle\eta_{\perp}\right\rangle$ is $11.8 \%$.
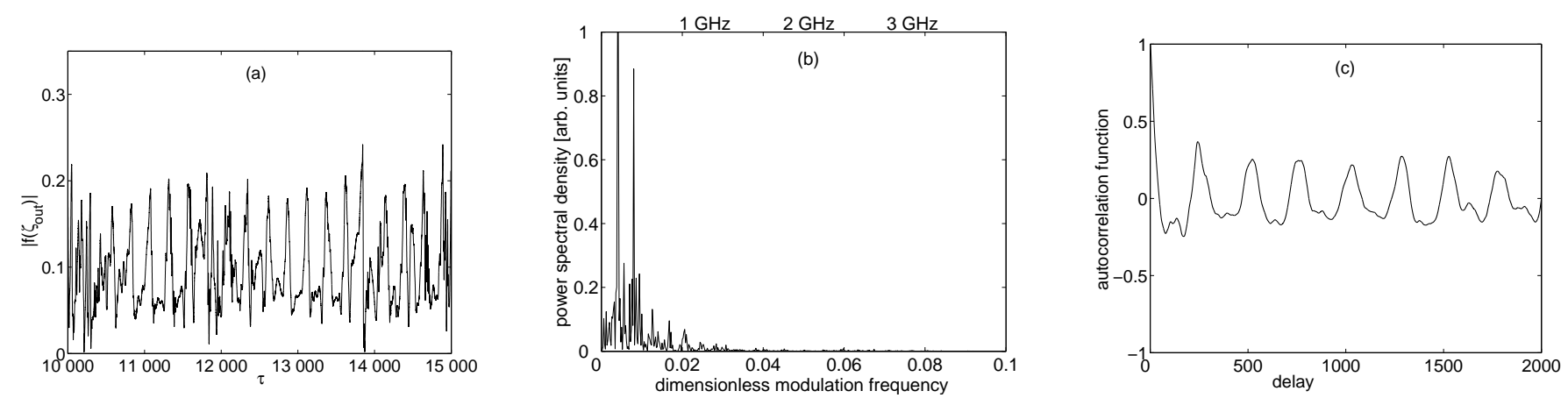

Fig. 3. (a) Gyrotron signal, (b) its power spectrum, and (c) autocorrelation function for $\Delta=0.20, I=0.10, R=0.30$, and $T=200$. Two dominant peaks appear in the spectrum in spite of the apparent irregularity of the signal. $\left\langle\eta_{\perp}\right\rangle=11.6 \%$.
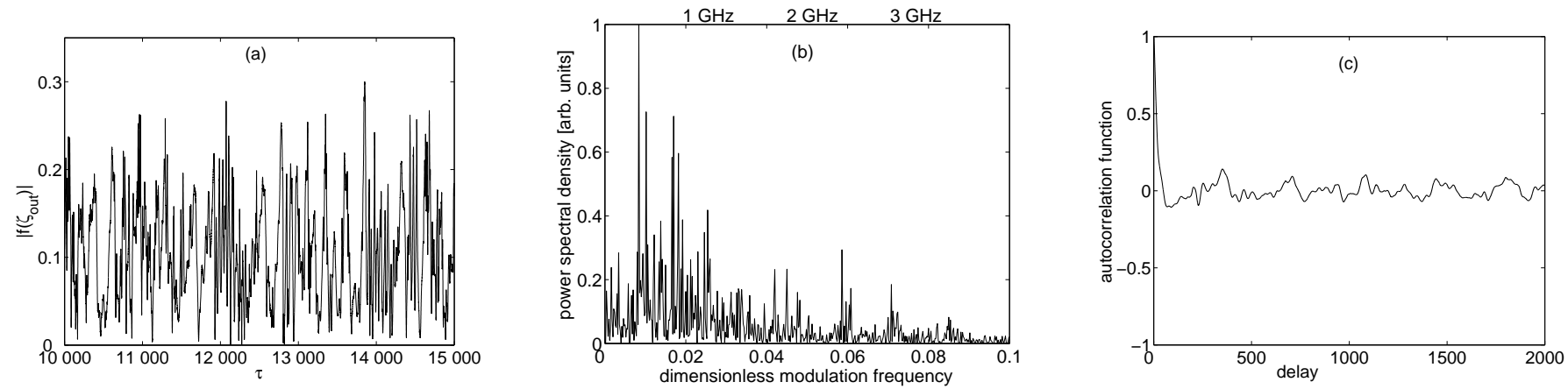

Fig. 4. (a) Gyrotron signal, (b) its power spectrum, and (c) autocorrelation function for $\Delta=0.20, I=0.10, R=0.45$, and $T=200$. The signal is chaotic with a broad spectrum and practically no autocorrelation. $\left\langle\eta_{\perp}\right\rangle=11.0 \%$.
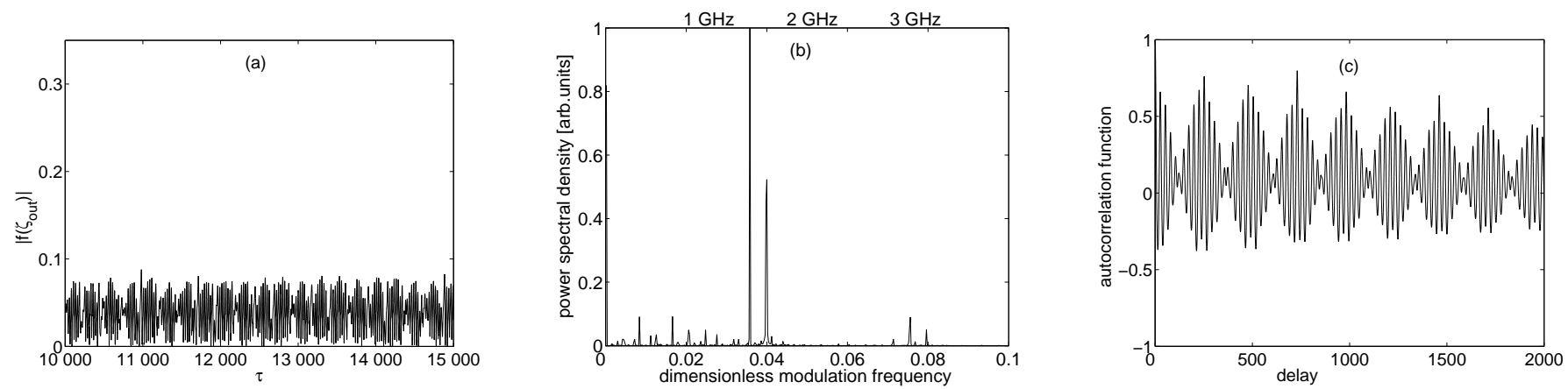

Fig. 5. (a) Gyrotron signal, (b) its power spectrum, and (c) autocorrelation function for $\Delta=0.0, I=0.018, R=0.45$, and $T=200$. Clearly separated frequency peaks reveal that the signal is quasiperiodic. 


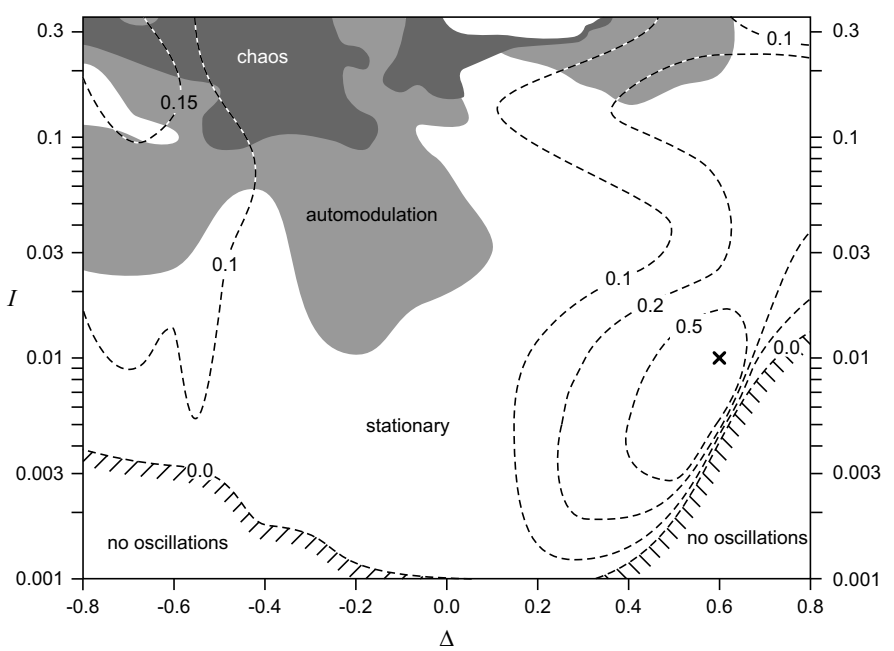

(a) No reflection

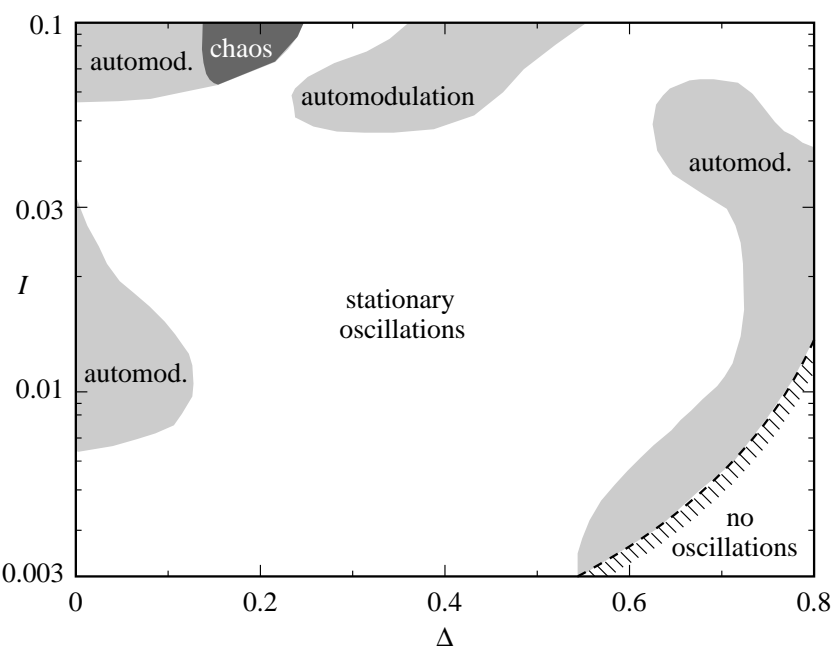

(b) $R=0.45, T=200$

Fig. 6. (a) Topology of different kinds of oscillations of a gyrotron in the $(\Delta, I)$ plane in the absence of reflections (Fig. 1 of [4]). The white region corresponds to stationary oscillations, gray regions correspond to automodulation, and dark regions to chaotic oscillations. (b) A part of the region shown in (a) with $R=0.45$ and $T=200$. Note that the parameter ranges differ.

\section{SUMmARY AND CONCLUSIONS}

We have presented a flexible formulation to describe reflections in the self-consistent time-dependent gyrotron theory and given the numerical algorithm needed for corresponding computations. The most important parameters characterizing reflection were concluded to be the absolute value of the reflection coefficient and the delay time. When stationary oscillations are considered, the reflection phase could be used instead of the delay time. The effect of the reflection phase on the stationary field profile, and consequently on generation efficiency, is rather strong. Using our description, reflections from distant objects can be simulated without remarkably slowing down the computations. However, the system itself attains its final state in a time scale that is much longer than the delay, so the computer runs must be accordingly extended in duration.

The breakdown of stationary oscillations to automodulation and further into chaotic oscillations was illustrated with a specific example and by recomputing the map of gyrotron oscillations presented in [4] with reflections included. It was found that new regions of nonstationary oscillations are generated and the existing ones shift to slightly lower currents due to reflection. Our results support the idea that reflection tends to drive a gyrotron into quasiperiodic rather than chaotic oscillations. Chaos appears in Fig. 4 just as a curiosity indicating small changes in the locations of the chaotic regimes in the parameter space.

It is interesting that automodulation was found for almost all current values when $0.6 \leq \Delta \leq 0.8$. This is in sharp contrast with the reflectionless case, where the high-efficiency region is surrounded by regimes of stationary oscillations only. Our findings can therefore be exploited in the development of high-power gyrotrons, which should provide a stationary signal even in the case of accidental reflections. By identifying in the operating parameter plane those regions where chaotic oscillations may be obtained, the results also ease the design of gyrotrons for applications which require broad bandwidth.

\section{ACKNOWLEDGMENTS}

We want to thank Prof. O. Dumbrajs and Dr. A. Grudiev for useful discussions and suggestions concerning the manuscript. Computing resources for this work were offered by the Finnish IT Center for Science.

\section{REFERENCES}

[1] N.S. Ginzburg, N.A. Zavolsky, G.S. Nusinovich, and A.S. Sergeev, "Self-oscillation in UHF generators with diffraction radiation output," Izv. Vyssh. Uchebn. Zaved. Radiofiz., vol. 29, pp. 106-114, Jan. 1986 [Sov. Radiophys. Quantum Electron., vol. 29, pp. 89-97, Jan. 1986].

[2] N.S. Ginzburg, G.S. Nusinovich, and N.A. Zavolsky, "Theory of nonstationary processes in gyrotrons with low $Q$ resonators," Int. J. Electron., vol. 61, pp. 881-894, Dec. 1986.

[3] N.S. Ginzburg, N.A. Zavolsky, and G.S. Nusinovich, "Dynamics of gyrotrons with a variable longitudinal structure of the microwave field," Radiotekh. Elektron., vol 32, 1987 [Sov. J. Commun. Technol. Electron., vol. 32, pp. 132-139, Sept. 1987].

[4] M.I. Airila, O. Dumbrajs, A. Reinfelds, and U. Strautiňš, "Nonstationary oscillations in gyrotrons," Phys. Plasmas, vol. 8, pp. 4608-4612, Oct. 2001

[5] T.H. Chang, S.H. Chen, L.R. Barnett, and K.R. Chu, "Characterization of stationary and nonstationary behavior in gyrotron oscillators," Phys. Rev. Lett., vol. 87, 064802, Aug. 2001.

[6] M.Yu. Glyavin and V.E. Zapevalov, "Reflections influence on the gyrotron oscillation regimes," Int. J. Infrared Millimeter Waves, vol. 19, pp. 1499-1511, Nov. 1998.

[7] N.S. Ginzburg, M.Yu. Glyavin, N.A. Zavolsky, V.E. Zapevalov, M.A. Moiseev, and Yu.V. Novozhilova, "A proposal to use reflection with delay for achieving the self-modulation and stochastic regimes in millimeter-wave gyrotrons," Pis'ma Zh. Tekh. Fiz., vol. 24, pp. 53-59, Jun. 1998 [Tech. Phys. Lett., vol. 24, pp. 436-438, Jun. 1998].

[8] O. Dumbrajs, M.Yu. Glyavin, V.E. Zapevalov, and N.A. Zavolsky, "Influence of reflections on mode competition in gyrotrons," IEEE Trans. Plasma Sci., vol. 28, pp. 588-596, Jun. 2000.

[9] M.I. Airila, O. Dumbrajs, P. Kåll, and B. Piosczyk, "Influence of reflections on the operation of the $2 \mathrm{MW}, \mathrm{CW} 170 \mathrm{GHz}$ coaxial cavity gyrotron for ITER," Nucl. Fusion, to be published.

[10] T.M. Antonsen, Jr., S.Y. Cai, and G.S. Nusinovich, "Effect of window reflection on gyrotron operation," Phys. Fluids B, vol. 4, pp. 4131-4139, Dec. 1992 
[11] A. Grudiev, J. Jelonnek, and K. Schünemann, "Time-domain analysis of reflections influence on gyrotron operation," Phys. Plasmas, vol. 8, pp. 2963-2973, Jun. 2001.

[12] A. Grudiev and K. Schünemann, "Nonstationary behavior of a gyrotron in the presence of reflections," Int. J. Infrared Millim. Waves, vol. 24, pp. 429-449, Apr. 2003.

[13] E. Borie, "Effect of reflection on gyrotron operation," IEEE Trans. Microwave Theory Tech., vol. 49, pp. 1342-1345, Jul. 2001.

[14] W.H. Press, S.A. Teukolsky, W.T. Vetterling, and B.P. Flannery, Numerical Recipes in FORTRAN. The Art of Scientific Computing (Cambridge University Press, New York, 1994), p. 43.

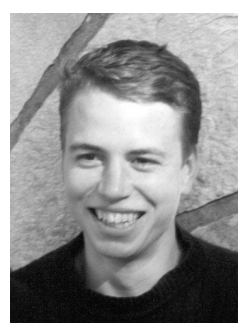

Markus Airila was born in Kerava, Finland, on March 16, 1975. He received the M.Sc. (Tech.) degree in engineering physics from Helsinki University of Technology, Espoo, Finland, in 2000. Currently, he is working towards the D.Sc. (Tech.) degree at the Helsinki University of Technology. His research interests include gyrotron theory, inverse problems in interferometry, and laser-plasma interactions.

Mr. Airila is a Member of the Finnish Physical Society and the Finnish Nuclear Society.

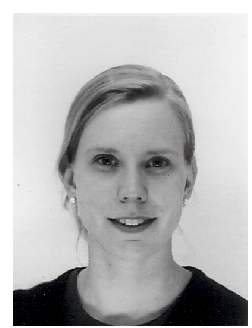

Pia Kåll was born in Porvoo, Finland, in 1980. She is working towards the M.Sc. (Tech.) degree in engineering physics at Helsinki University of Technology, Espoo, Finland, where she also currently participates in the development of ELMFIRE, a gyrokinetic particle code for fusion plasma simulations. 